\title{
An offshore reservoir monitoring system based on fibre-optic distributed sensing of seabed strains
}

\section{Levenberg, Eyal; Orozova-Bekkevold, Ivanka}

\section{Published in:}

First Break

Link to article, DOI:

10.3997/1365-2397.fb2020016

Publication date:

2020

Document Version

Early version, also known as pre-print

Link back to DTU Orbit

Citation (APA):

Levenberg, E., \& Orozova-Bekkevold, I. (2020). An offshore reservoir monitoring system based on fibre-optic distributed sensing of seabed strains. First Break, 38(3), 35-41. https://doi.org/10.3997/1365-2397.fb2020016

\section{General rights}

Copyright and moral rights for the publications made accessible in the public portal are retained by the authors and/or other copyright owners and it is a condition of accessing publications that users recognise and abide by the legal requirements associated with these rights.

- Users may download and print one copy of any publication from the public portal for the purpose of private study or research.

- You may not further distribute the material or use it for any profit-making activity or commercial gain

- You may freely distribute the URL identifying the publication in the public portal 


\title{
An offshore reservoir monitoring system based on fibre-optic distributed sensing of seabed strains
}

\author{
Eyal Levenberg and Ivanka Orozova-Bekkevold
}

\begin{abstract}
One of the problems affecting mature hydrocarbon fields, e.g., Ekofisk, Tyra, and Dan in the North Sea, is seabed subsidence due to reservoir depletion. Fluid injection is a widely used method to boost production and/or maintain reservoir pressure in order to mitigate subsidence. Both reservoir depletion and fluid injection operations induce seabed deformations. The deformation pattern potentially holds useful information about production efficiency and reservoir management, which could be captured by careful monitoring of seabed strains. Therefore, the idea suggested herein was performing near full-field and continuous monitoring of seabed deformations by means of distributed fibre-optic strain sensing. The objective of the study was to theoretically calculate and assess whether current technologies (i.e., off-the-shelf optical interrogators) are accurate and sensitive enough to detect production-induced seabed strains originating at a two-kilometre deep reservoir. The analysis indicates that depletion-induced subsidence is potentially measurable with seabed distributed fibreoptic strain sensing. However, the operation of an injector-producer array induces minute seabed strains that are too small to be detected with current capabilities.
\end{abstract}




\section{Introduction}

Many of the Danish gas fields in the North Sea are produced by natural depletion with gas/fluid expulsion and related compaction as drivers (Hatchell et al. 2007; Danish Energy Agency 2013; Calvert et al. 2014; 2016; Schutjens et al. 2019). Often, reservoir compaction is translated into seabed subsidence, which has a direct adverse effect on production facilities. In the case of the Tyra gas field, for example, the maximum observed subsidence due to production reached approximately five metres (Schutjens et al. 2019). To a lesser extent, seabed subsidence is also observed in oil fields such as Dan (Hatchell et al. 2007). Initially, the Dan field was produced by natural depletion - with water injection initiated only after several years of production. Analysis of timeshift data from the 4D seismic surveys acquired in the Halfdan/Dan field in 2005 and 2012 (Calvert et al. 2014; 2016) indicated that waterflooding might have slowed down reservoir compaction in the area. Nonetheless, albeit reduced, seabed subsidence has continued - suggesting that the compaction drive remained active. Modern developments, such as the Halfdan Field (Danish Energy Agency 2013), have parallel injection and producer wells resulting in near-zero seabed subsidence (Calvert et al. 2014; 2016). Nevertheless, pressure differences between injector and producer wells may have caused minor deformations of the seabed - leading to minor positive and negative vertical displacements at the different Halfdan platforms. It is plausible that loss of pressure support caused by short circuits between injector and production wells might have been the reason for the observed deformations (Hatchell et al. 2007). To the best of our knowledge, these small displacement ripples are difficult to capture by seismic data and have yet to be thoroughly investigated.

The idea advocated herein is to develop a reservoir monitoring system based on reading seabed deformations. This is to be achieved by means of distributed fibre-optic strain sensing technology, with fibre-optic cables coupled to the seabed. The idea is conceptually shown in Figure 1(a), which sketches an overhead view of a $12 \mathrm{~km} \times 12 \mathrm{~km}$ production field that is instrumented with a single fibre-optic cable deployed around a platform. The shown meshing is geared towards reading both radial and tangential strains with respect to a cylindrical coordinate system with origin at the bottom of the platform. In this arrangement, redundancy is assured by fibre-optic cable loops that periodically return to the platform. Thus, any accidentally damaged loop can be skipped by means of splicing operations taking place on the platform - without the need for expensive underwater gear. In practice, the deployed pattern should be tailored to the production setup with fibre orientations corresponding to the anticipated principal strain directions. Figure 1(b) shows a seabed trencher; a semi-robotic device that can potentially deploy a fibre-optic cable according to a desired pattern (Fugro Inc. 2017). This device can bury a cable at a depth of about two metres below the seabed, thus ensuring mechanical coupling with the surrounding soil medium. Such burial depth also provides protection against fishing trawls as well as isolation from storm effects. Finally, Figure 1(c) illustrates how fibreoptic cables can be brought to the platform deck through a so-called J-tube (Bai \& Bai 2005) in order 
to be connected to an optical interrogator - which typically consists of a delicate laser source and receiver optics.

(a)

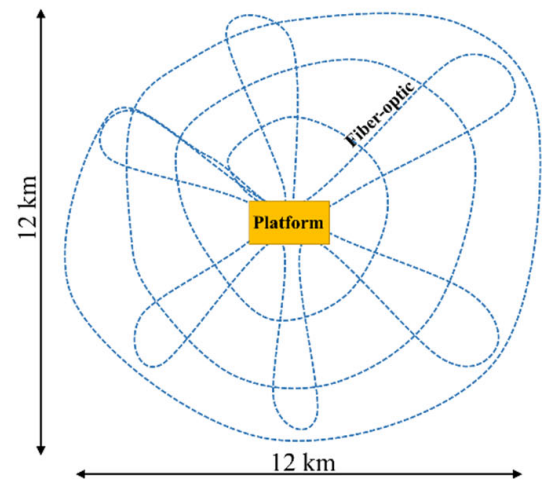

(b)

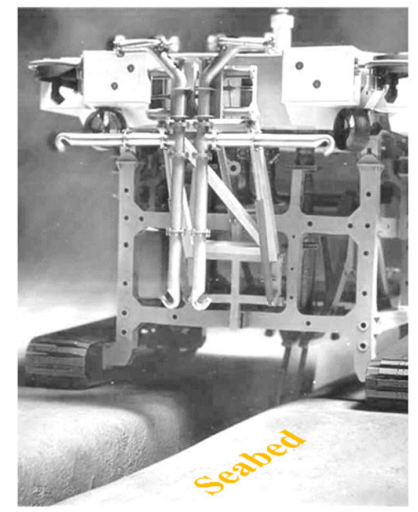

(c)

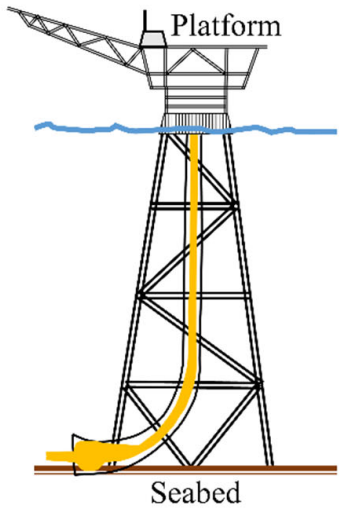

Figure 1: Practical deployment of a distributed fibre-optic sensing array; (a) possible deployment pattern around an offshore platform; (b) seabed trencher for sensor embedment (Fugro Inc. 2017); and (c) J-tube for bringing fibre-optic cables onto the platform.

Distributed fibre-optic strain sensing is an established technology, commonly employed for monitoring Civil Engineering facilities (Barrias et al. 2016). Fibre-optic sensors have also been employed in the oil and gas industry, as point strain sensors (Yanlin et al. 2011) or along pipes (Inaudi \& Glisic 2010). A recent survey of fibre-optic sensing applications within the oil and gas industry can be found in Daniel et al. (2017). Ideally, the monitoring system should be deployed and activated before the start of production. For already producing fields, such as in the North Sea, a fibre-optic monitoring system deployed after the start of production will sense only events/changes that take effect subsequent to installation.

Whether the system is installed pre- or post-production start, it offers several potential benefits: (i) help in monitoring and prediction of reservoir compaction and related subsidence, (ii) provide indication on possible location of short circuits between injection and production wells, (iii) monitor and/or predict the changes in the gap between the deck of the platforms and the sea surface, (iv) provide additional input to 4D seismic surveys, (v) monitor potential smart water that can increase the compaction drive, (vi) monitor induced seismicity due to production activities, and (vii) monitor post-production deformations to accommodate any future environmental enquiries by the authorities. These benefits can lead to better decisions on production, re-development, and end-of-life strategies. By its very nature, the proposed system has an advantage over pipeline surveys which provide only information along a line, with readings that are susceptible to pipe corrosion, pipe failure, submarine storms, etc. Furthermore, when a more reliable technology arises, capable of reading fibre strains at 
a very fast rate of the order of $10 \mathrm{kHz}$, the deployed cables can be utilized as geophones. In this case, considerable price reduction in acquiring 4D seismic data is anticipated (Maas \& Buchan 2007).

Distributed fibre-optic strain sensing is based on the so-called Brillouin scattering effect wherein the light intensity in the fibre locally modifies the density of the solid, resulting in scattering and frequency shift (Galindez-Jamioy \& López-Higuera 2012). Optical detection systems currently available on the market for distributed strain sensing operate with standard telecommunications optical fibres. The maximum sensing length for these systems is about $100 \mathrm{~km}$ (fibre length can be longer) with a strain resolution of 0.1 microstrains and accuracy of \pm 2 microstrains. If desired, the spatial resolution can be as short as $5 \mathrm{~cm}$. The optical interrogator devices are of the size scale of desktop computers.

\section{Objective and methodology}

The main objective of the current work is to carry out an in silico feasibility study that investigates whether current distributed fibre-optic strain sensing technologies can offer sufficient resolution and accuracy to detect production-induced seabed strains. Such assessment is a first and necessary step before any further development because the associated strains are expected to be very small. An existing analytic theory for computing mechanical responses due to deep deformations is employed as a basic modelling tool (Levenberg 2013). As shown in Figure 2, the theory considers a laterally infinite, weightless, isotropic, homogeneous, and linear elastic layer with thickness $H$ and material properties $E$ (Young's modulus) and $v$ (Poisson's ratio). Herein, this layer represents the overburden, i.e., the entire strata overlaying a reservoir. Initially, the medium is assumed to be undeformed and stress-free, with both top and bottom boundaries completely flat (dash-dot lines), i.e., any existing stresses due to self-weight are taken as initial conditions and therefore annulled. Next, an axisymmetric blister-like displacement field is imposed at the bottom of the layer, forcing the lower boundary to deform vertically, without inducing shear stresses at the interface (solid line).

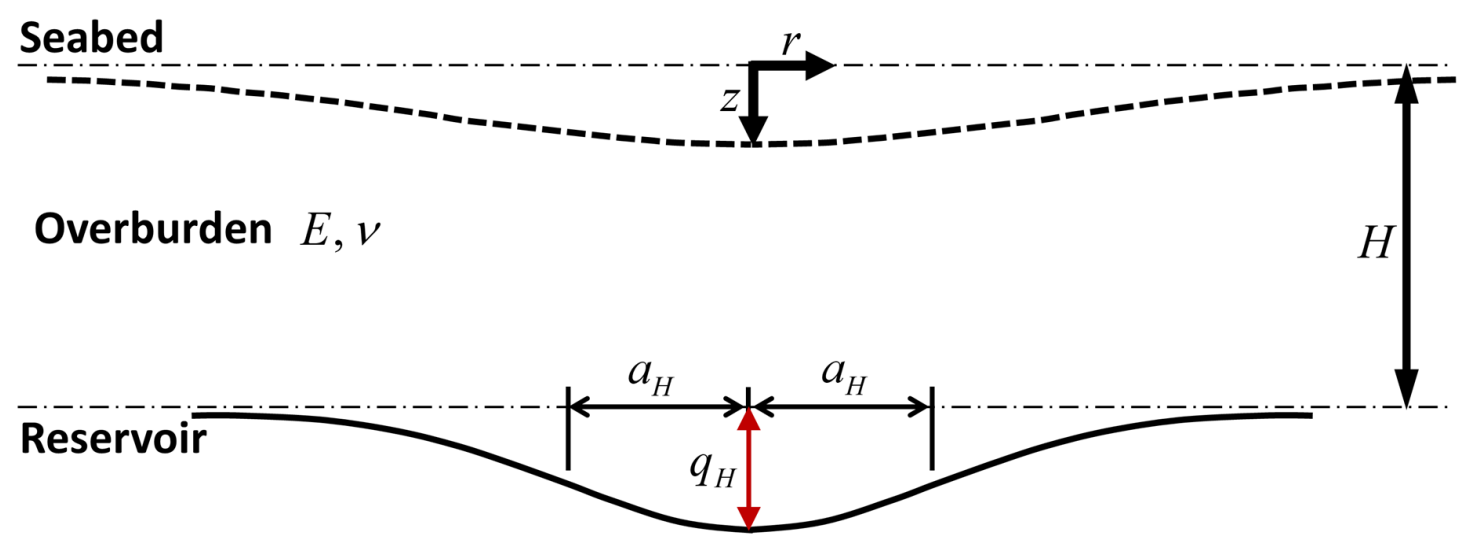

Figure 2: Illustration of basic model definitions. 
The modelling allows for the calculation of all mechanical responses (i.e., stresses, strains, and displacements) inside the layer as well as at the top, corresponding to the seabed level. To facilitate the formulation, a cylindrical coordinate system is included (see Figure 2), with its origin placed at the top of the undeformed layer, the depth $z$-axis drawn into the medium, and the radial $r$ -axis $(r \geq 0)$ parallel to the top boundary. The imposed displacement field at bottom is denoted by $u_{z}^{H}$, mathematically expressed as an axisymmetric Gaussian:

$$
u_{z}^{H}(r)=q_{H} e^{-\left(r / a_{H}\right)^{2} / 2}
$$

where $r$ is the radial coordinate, $q_{H}$ is the peak displacement occurring at $r=0$, and $a_{H}$ represents the location where the curvature of $u_{z}^{H}(r)$ changes sign (i.e., inflection point). Both $q_{H}$ and $a_{H}$ have units of length; $a_{H}$ must be positive, while $q_{H}$ can be either positive or negative to indicate downward or upward deformation (respectively). The particular form of Equation 1 was chosen because it can serve as a radial basis function for representing any deformation shape - not necessarily axisymmetric (Goshtasby \& O’Neill 1993). This modelling flexibility is needed when simulating, e.g., the effects of a producer-injector array.

The mechanical responses at the top of the layer (where $z=0$ ) are calculated from the following equations:

$$
\begin{aligned}
& \sigma_{r}^{0}=-E \int_{m=0}^{\infty}\left(\left(m J_{0}(m \bar{r})-\frac{J_{1}(m \bar{r})}{\bar{r}}\right)\left(\begin{array}{l}
(A+C) e^{-m} \\
+B-D
\end{array}\right)+2 v m J_{0}(m \bar{r})\left(C e^{-m}-D\right)\right) d m \\
& \sigma_{\theta}^{0}=-E \int_{m=0}^{\infty}\left(\frac{J_{1}(m \bar{r})}{\bar{r}}\left(A e^{-m}+C e^{-m}+B-D\right)+2 v m J_{0}(m \bar{r})\left(C e^{-m}-D\right)\right) d m \\
& u_{z}^{0}=-H(1+v) \int_{m=0}^{\infty}\left(J_{0}(m \bar{r})\left(A e^{-m}-C(2-4 v) e^{-m}-B-D(2-4 v)\right)\right) d m \\
& u_{r}^{0}=H(1+v) \int_{m=0}^{\infty}\left(J_{1}(m \bar{r})\left(A e^{-m}+C e^{-m}+B-D\right)\right) d m
\end{aligned}
$$

where $\sigma_{r}^{0}, \sigma_{\theta}^{0}, u_{z}^{0}$, and $u_{r}^{0}$ denote (respectively) radial stress, tangential stress, vertical displacement, and radial displacement; $m$ is a unitless integration parameter. The superscript zero indicates that these responses are calculated at $z=0$ (seabed). Moreover, $\bar{r}=r / H$ is a normalized (dimensionless) radial coordinate, $J_{0}(\cdot)$ and $J_{1}(\cdot)$ are Bessel functions of the first kind of order zero and one (respectively), and $A, B, C$, and $D$ are each a dimensionless function of $m$. The latter are obtained from enforcing the problem boundary conditions; they are explicitly listed in Levenberg (2015). 
Seabed strains, expected to be read by the fibre-optic sensors, are derived from the stresses in the usual way:

$$
\begin{aligned}
& \varepsilon_{r}^{0}=\frac{\sigma_{r}^{0}-v \sigma_{\theta}^{0}}{E} \\
& \varepsilon_{\theta}^{0}=\frac{\sigma_{\theta}^{0}-v \sigma_{r}^{0}}{E}
\end{aligned}
$$

where $\varepsilon_{r}^{0}$ and $\varepsilon_{\theta}^{0}$ denote the strain in the radial direction and tangential direction (respectively). Note that these strains are not affected by the overburden modulus because it cancels-out when inserting the stress expressions from Equations 2 and 3.

\section{Simulation and analysis}

Two scenarios are considered hereafter; the first deals with simulating the conditions of depletioninduced subsidence, for example in the case of gas production. The second deals with monitoring a stimulated field consisting of an array of producers and injectors. For both cases it is assumed that the strain monitoring system is deployed and activated at some point in time after the start of production operations, and therefore detects only subsequent events.

\subsection{Depletion-induced subsidence}

The above theory is first utilized for investigating subsidence over a large area due to depletion. The overall displacement at top reservoir (i.e., bottom of the overburden) is idealized as being axisymmetric according to Equation 1. The assumed model parameters are: (i) overburden thickness $H=2000 \mathrm{~m}$, (ii) Poisson's ratio of medium $v=0.26$, (iii) width of Gaussian - representing displacement pattern at the reservoir top $a_{H}=1000 \mathrm{~m}$, and (iv) an assumed peak reservoir compaction $q_{H}=300 \mathrm{~mm}$ - roughly representing the detection resolution of current technologies for monitoring reservoir subsidence.

Model calculation results are shown in Figures 3 and 4. Figure 3(c) and 3(d) present the vertical displacement fields at top reservoir (i.e., $u_{z}^{H}$ ), while Figure 3(a) and 3(b) describe the vertical displacement field at the seabed level (i.e., $u_{z}^{0}$ ). It can be seen that the induced displacement is attenuated at the seabed. The peak vertical displacement drops from $300 \mathrm{~mm}$ (reservoir top) to about $180 \mathrm{~mm}$ at seabed (overburden top). Also, the deformation pattern, i.e., the subsidence bowl, is somewhat wider at the surface. Figures 4(a) and 4(b) present the corresponding seabed strains in the radial direction, while Figures 4(c) and 4(d) depict the tangential strain distribution. It can be seen that radial strains $\left(\varepsilon_{r}^{0}\right)$ change sign from positive (compression) to negative (tension) while the tangential strains $\left(\varepsilon_{\theta}^{0}\right)$ are only compressive. The strain levels in both directions are of the order of 
50 microstrains, i.e., within the resolution and accuracy limits of current distributed fibre-optic strain sensing technologies.
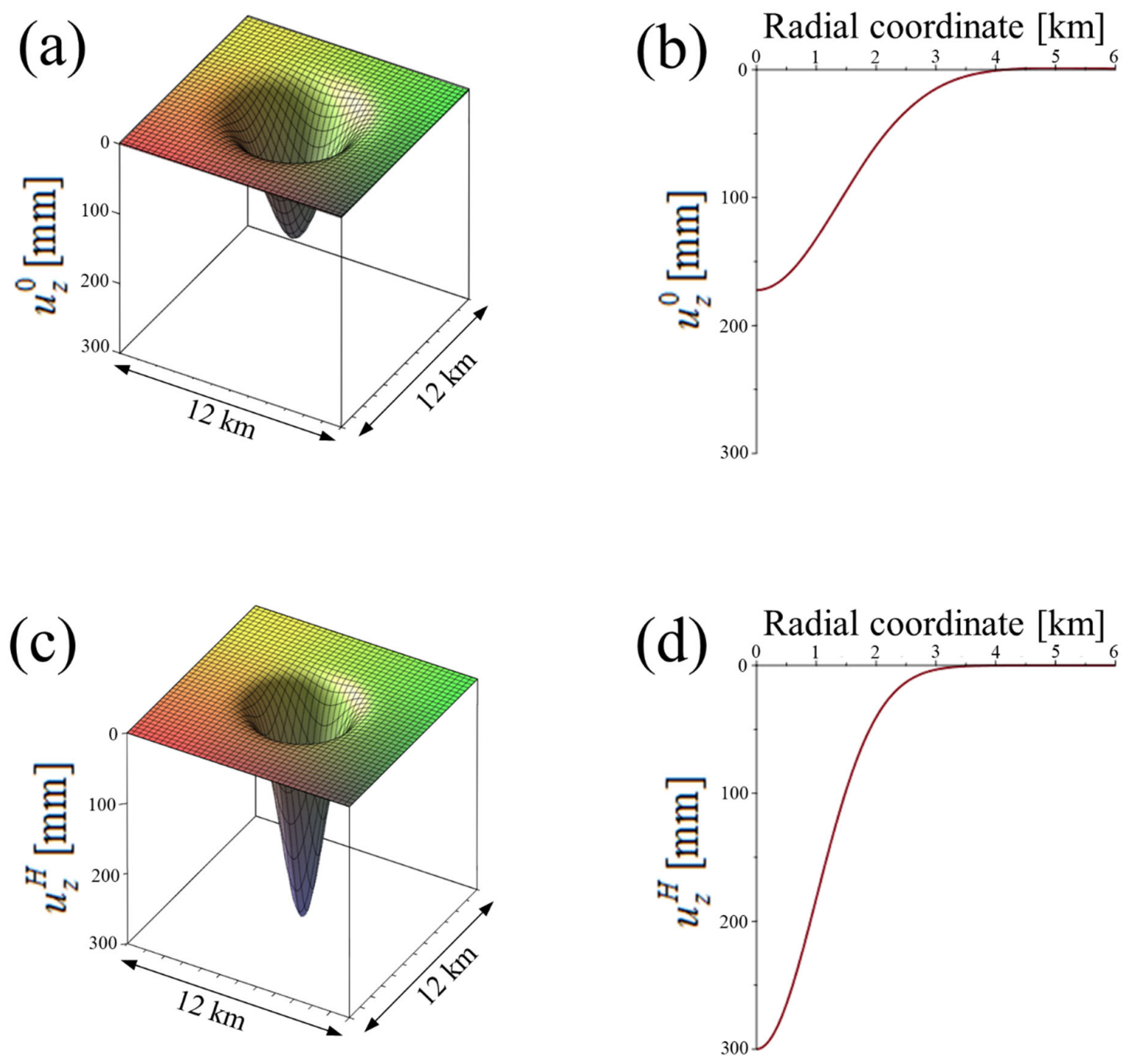

Figure 3: Vertical displacement field in the axisymmetric case; (a) and (b) displacements at seabed level, and (c) and (d) displacements at reservoir level. 

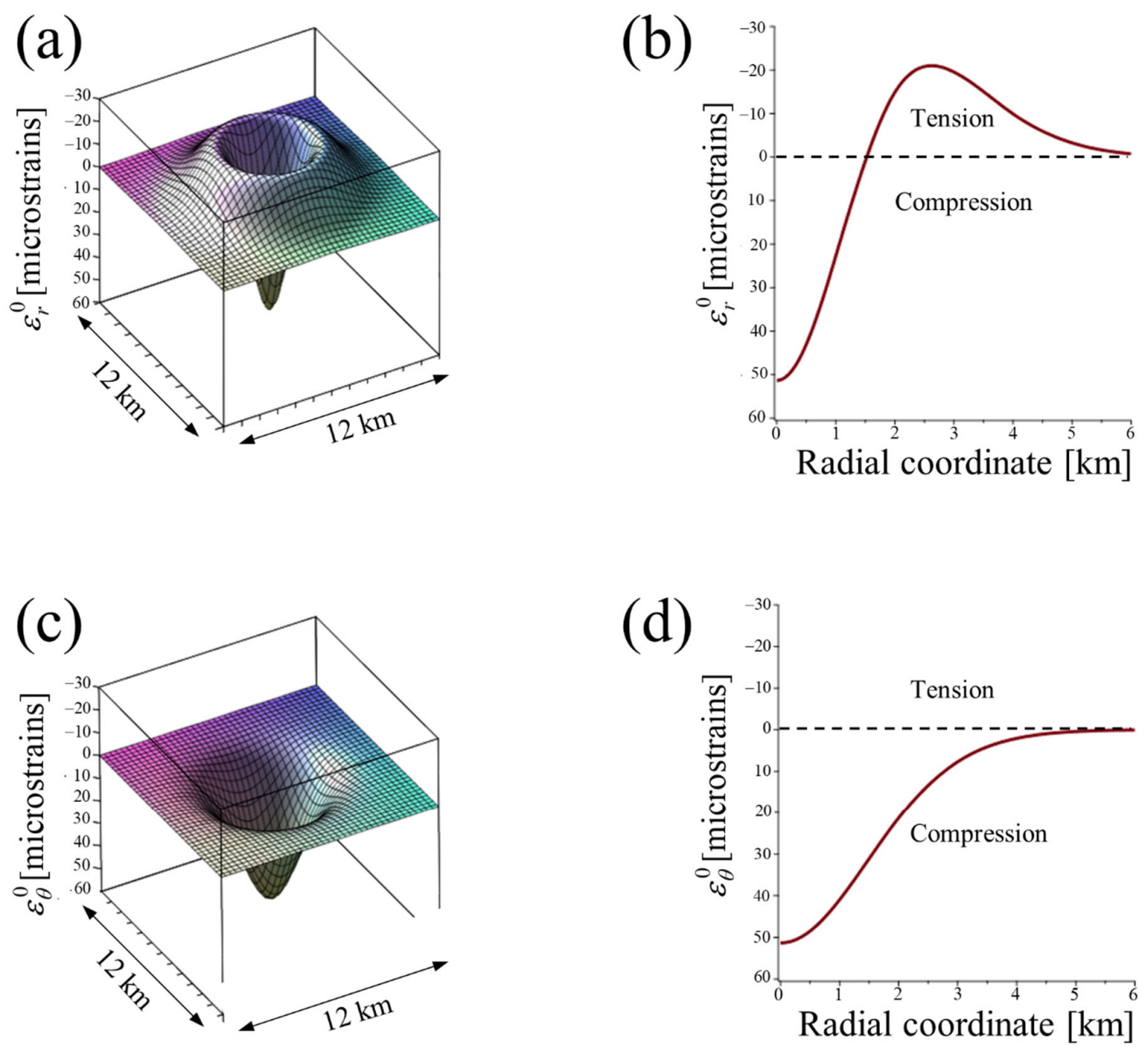

Figure 4: Seabed strain distributions in the axisymmetric case; (a) and (b) radial strains, and (c) and (d) tangential strains.

\subsection{An injector-producer array}

The second considered case dealt with a virtual injector-producer array consisting of three producers, denoted as $P_{1} \ldots P_{3}$, and four injectors, denoted as $I_{1} \ldots I_{4}$. The array is arranged as shown in Figure 5(a) with $L$ denoting length and $W$ denoting the spacing between injectors and producers. A Cartesian coordinate system is employed in this case, with the $y$-axis oriented parallel to the wells, the $x$-axis pointing in the transverse direction, and the $z$-axis (not shown) pointing downward towards the earth's core. A proposed pattern for fibre-optic deployment, matching the geometry of the injectorproducer array and the expected principal strain directions, is shown in Figure 5(b). As can be seen, the pattern includes several loops, generally oriented in the directions of the $x$ and $y$ axes. As in 
Figure 1, redundancy and ease of maintenance is enabled by periodically returning the fibre to the platform to allow for splicing in case of an accidentally damaged loop.

(a)

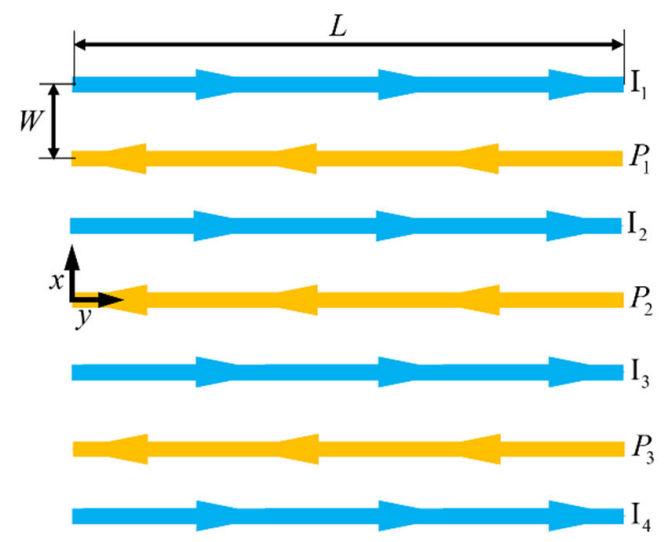

(b)

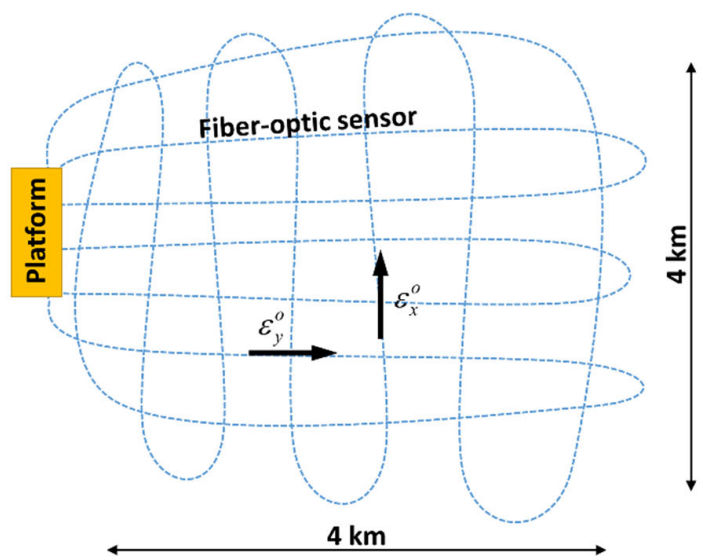

Figure 5: (a) a virtual injector-producer array; and (b) a possible fibre-optic deployment pattern at seabed level.

Under operation, the injector-producer array in Figure 5 imposes vertical deformations at the bottom of the overburden (top reservoir) - depression in the vicinity of the producers and heaving in the vicinity of the injectors. Such deformation pattern can be approximated mathematically by the following expression:

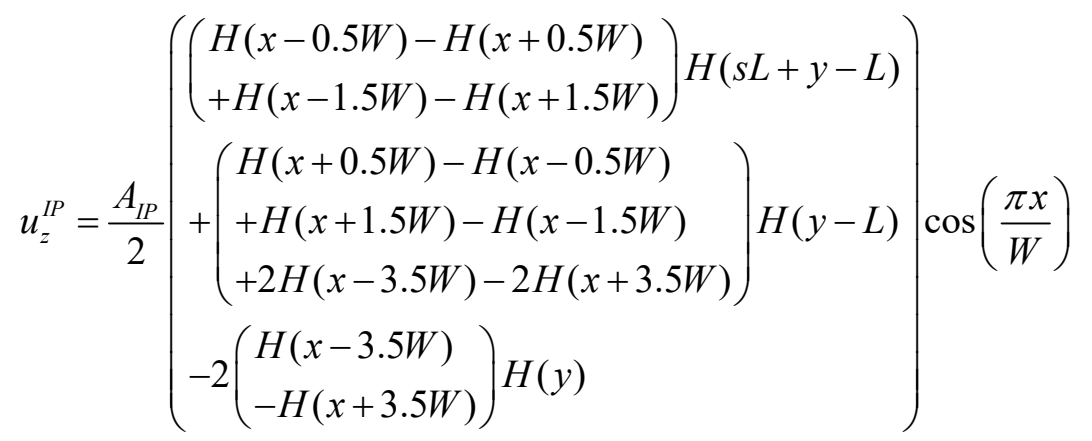

where $u_{z}^{I P}$ is the vertical displacement magnitude at the reservoir level at an arbitrary location/point identified by coordinates $x$ and $y$. The parameter $A_{I P}$ denotes the displacement amplitude induced by either an injector or a producer, and $H(\cdot)$ is the Heaviside step function. The parameter $s$ can range between $0 \%$ and $100 \%$; it is introduced to represent a shortcut event between injector wells $I_{2}$ and $I_{3}$. If this condition occurs, the heave amplitude caused by $I_{2}$ and $I_{3}$ is reduced by $50 \%$ along a length of $s L$. At the same time, the depression along $P_{2}$ becomes limited to a length $L(1-\mathrm{s})$. In other words, when $s=0 \%$ the injector-producer array is fully operational, and when $s>0 \%$ a 
partially operational situation is simulated. A full shortcut between $I_{2}$ and $I_{3}$ is simulated when $s=100 \%$.

Assuming that a strain monitoring system is installed at the seabed when the array is fully functional ( $s=0 \%$ condition), subsequent strain readings will reflect deformations caused by any shortcut event, i.e., the difference between $u_{z}^{I P}(s>0 \%)$ and $u_{z}^{I P}(s=0 \%)$. This situation is illustrated in Figure 6 where the top three images, Figure 6(a), (b), and (c), provide plots of $u_{z}^{I P}$ for different $s$ values. The bottom three images, Figure 6(d), (e), and (f) depict the corresponding displacements (at top reservoir) that generate strains at the seabed level. The common parameters for preparing the images were: $W=200 \mathrm{~m}, L=2 \mathrm{~km}$ and $A_{I P}=1 \mathrm{~m}$. The choice for $A_{I P}=1 \mathrm{~m}$ should be a subject of further study given that the actual peak displacement magnitude near the injectors and producers is not known. The vertical axes in Figure 6 images are graphically distorted $(\times 50)$, so that the deformation pattern can be noticed.

Figure 7 shows the estimated seabed strains corresponding to the three cases in Figure 6 (bottom three charts), i.e., for the three different $s$ values: 25\%, 50\%, and 75\%. Figures 7(a), (b) and (c) depict seabed strains along the $x$-axis direction $\left(\varepsilon_{x}^{0}\right)$ and Figures $7(\mathrm{~d}),(\mathrm{e})$, and (f) depict seabed strains along the $y$-axis direction $\left(\varepsilon_{y}^{0}\right)$. To arrive at these strains the induced deformation shapes were first re-written, separately in each case, as a sum of more than 300 Gaussians (with a priori chosen coordinates and equal width); each Gaussian had a different peak displacement obtained by a means of a collocation technique. Then, the above described axisymmetric theory (specifically, Equations 6 and 7) was applied to obtain individual strain solutions. Finally, given the linearity of the modelling, these strain solutions were superposed to yield the results presented in Figure 7. As it can be seen, the peak-to-peak strain magnitudes are of the order of a few tenths of a microstrain. These strain levels are similar in magnitude to the current resolution limits of distributed fibre-optic sensing (about 0.1 microstrains), and about an order of magnitude smaller than the accuracy limits of the technology (about \pm 2 microstrains). 

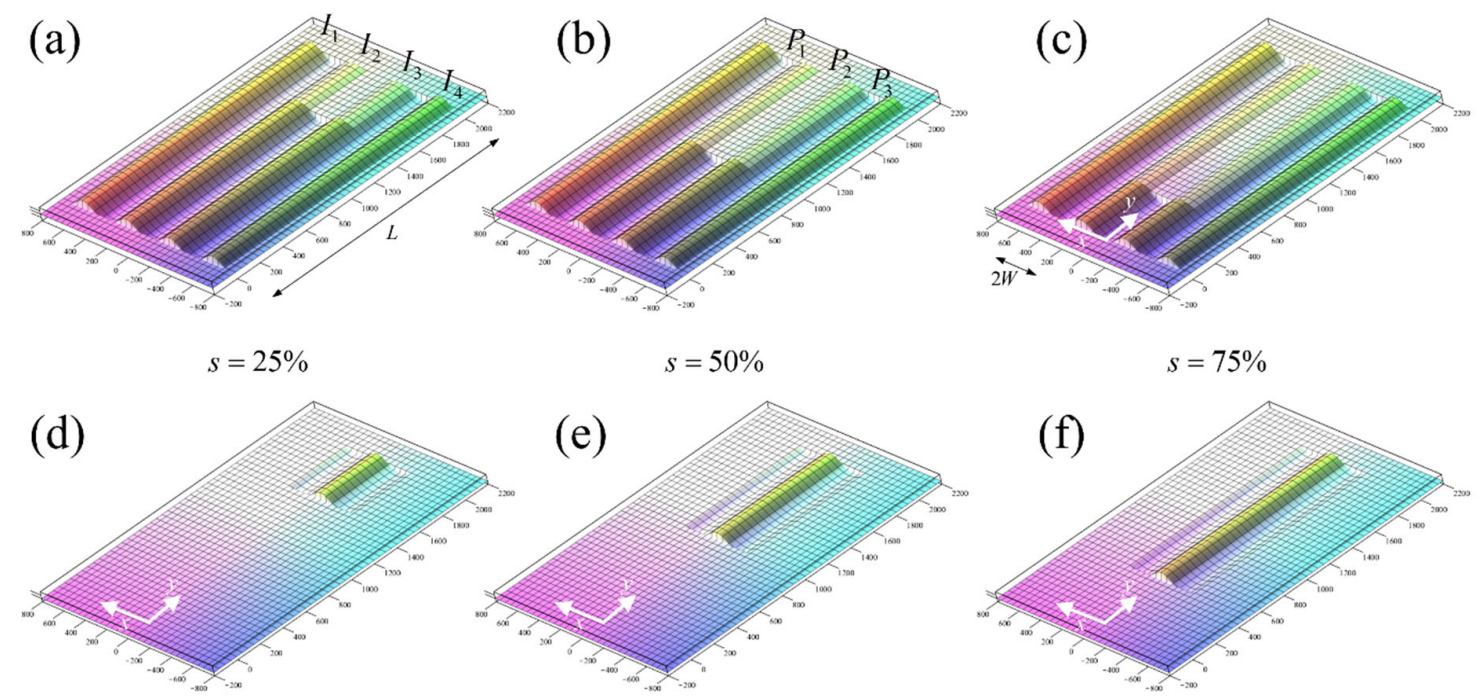

Figure 6: Simulating a partially-operational Injector-Producer array (refer to Figure 5); (a), (b), and (c) represent the assumed displacement pattern for different shortcut lengths (expressed via the $s$ parameter) according to Equation 7; (d), (e), and (f) display the corresponding displacement differences.

(a)
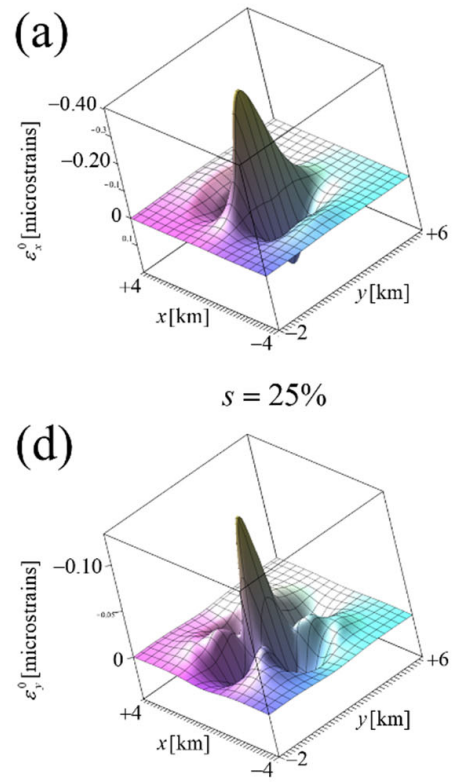
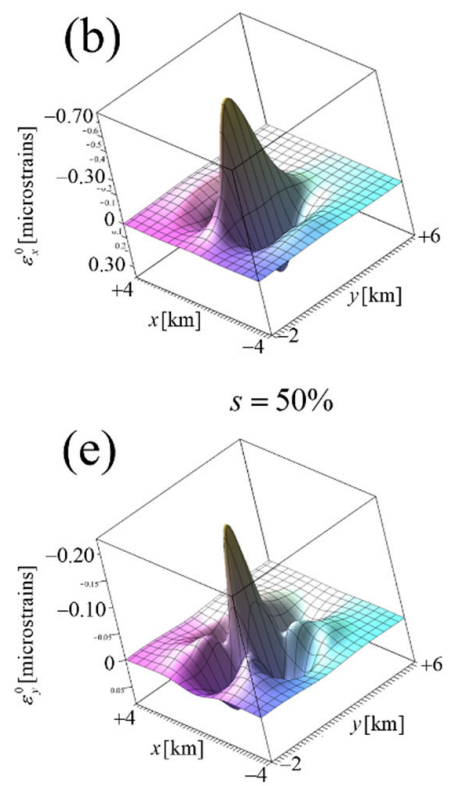
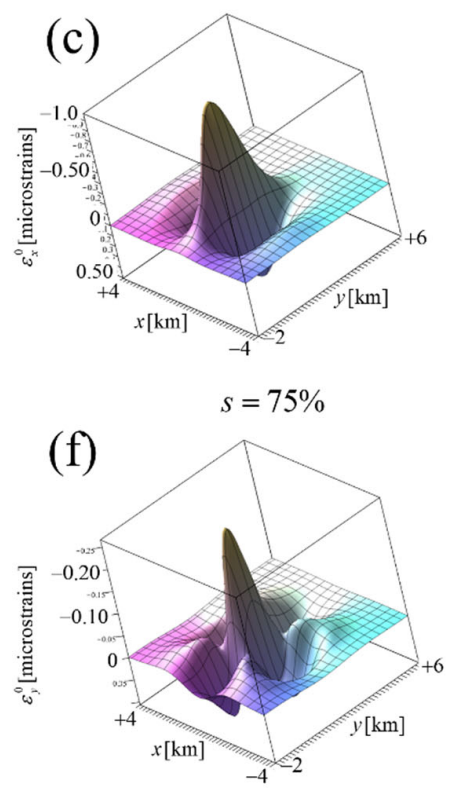

Figure 7: Calculated seabed strains in the $x$ and $y$ directions (see coordinates in Figures 5 and 6) corresponding to three different shortcut cases (d), (e), and (f) in Figure 6.

\section{Conclusion}

This work considered the idea of meshing the seabed near offshore platforms with fibre-optic sensors as a means of detecting deformations originating at the reservoir level. An analytic model was 
employed to estimate the magnitude and orientation of expected strains as a first-step feasibility study. Two separate cases were investigated: (i) monitoring the annual compaction of an entire field (see Figure 1), and (ii) monitoring the effects of shortcuts in an injector-producer array (see Figure 5). Based on the calculations, is it concluded that the expected seabed strain levels in the first case are potentially detectable and measurable with current distributed fibre-optic sensing technology, while the strain levels in the second case are very small, below the resolution and accuracy limits of available optical interrogators.

The idea proposed in this study is deemed promising, and it is therefore suggested to carry out further research to promote its realization. First and foremost it is recommended to improve the modelling: (i) by incorporating actual and more realistic and/or detailed production data and compaction/subsidence measurements; (ii) by considering a stratified overburden; and (iii) by considering more advanced material behaviour e.g., time-dependent properties and permanent deformation properties. Second, it may be relevant to construct a scaled-down controlled field experiment where optic fibres are physically deployed, and a buried actuator mechanically simulates reservoir displacements. Finally, it is suggested to develop an inverse analysis scheme that continuously accepts as input fibre-optic strain readings, and provides (as output) real-time information about the reservoir performance.

\section{Acknowledgment}

This paper is based on work carried out by the authors as part of the 2017 Radical Innovation Sprint Project initiated at the Centre for Oil and Gas - DTU / The Danish Hydrocarbon Research and Technology Centre (DHRTC). The authors would like to thank the DHRTC, and in particular Lene Hjelm Poulsen and Hans Horikx for their support and guidance.

\section{References}

Bai, Y. and Bai, Q. [2005] Subsea pipelines and risers. Elsevier, Amsterdam.

Barrias, A., Casas, J.R., and Villalba, S. [2016] A review of distributed optical fibre sensors for civil engineering applications. Sensors, 16(748), 1-35.

Calvert, M.A, Vagg, L.D., Lafond, K.B., Hoover, A.R., Ooi, K.C., and Herbert, I.H. [2014] Insights into sweep efficiency using 4D seismic at Halfdan field in the North Sea. The Leading Edge, 33(2), $182-187$.

Calvert, M.A, Vagg, L.D., Lafond, K.B., Hoover, A.R., Ooi, K.C., and Herbert, I.H. [2016] Halfdan 4D workflow and results leading to increased recovery. The Leading Edge, 35(10), 840-848.

Daniel, J.S., Maida, J.L., and Skinner, N.G. [2017] Adapting optical technology to dynamic energy prices: fibre-optic sensing in the contemporary oil field. SPIE Proceedings Vol. 10208: Fibre Optic Sensors and Applications XIV, Anaheim, California, United States. 
Danish Energy Agency [2013] Oil and gas production in Denmark (and subsoil use). Online report: https://ens.dk/sites/ens.dk/files/OlieGas/oil_and_gas_in_denmark_2013.pdf, accessed November 20, 2017.

Fugro Inc. TSM Q1400 Trenching System. Online link: youtube.com/watch?time_continue $=7 \& v=c F x l w W P m 4 f 0$, accessed November 20, 2017.

Galindez-Jamioy, C.A. and López-Higuera, J.M. [2012] Brillouin distributed fibre sensors: an overview and applications," Journal of Sensors, 2012, Article ID 204121 (DOI: $10.1155 / 2012 / 204121)$.

Goshtasby, A. and O’Neill, W.D. [1993] Surface fitting to scattered data by a sum of Gaussians. Computer Aided Geometric Design, 10(2), 143-156.

Hatchell, P.J., Jorgensen, O., Gommesen, L., and Stammeijer, J. [2007] Monitoring reservoir compaction from subsidence and time-lapse time shifts in the Dan field. Society of Exploration Geophysicists (SEG), Technical Program Expanded Abstracts 2007, 2867-2871 (DOI:10.1190/1.2793062).

Inaudi, D. and Glisic, B. [2010] Long-range pipeline monitoring by distributed fibre optic sensing. Journal of Pressure Vessel Technology, 132(1), 011701(1)-011701(9).

Levenberg, E. [2013] Analysis of pavement response to subsurface deformations. Computers and Geotechnics, 50, 79-88.

Levenberg, E. [2015] Intrinsic roughness mitigation of pavements on expansive soils - an analytic investigation. International Journal of Pavement Research and Technology, 8(3), 167-171.

Maas, S.J. and Buchan, I. [2007] Fibre optic 4C seabed cable for permanent reservoir monitoring. Proceedings of the International Symposium on Underwater Technology and Workshop on Scientific use of Submarine Cables Related Technologies, Tokyo, Japan, 411-414.

Schutjens, P.M.T.M., Fokker, P., Rai, U.B.B. et al. [2019] Compaction- and shear-induced well deformation in Tyra: Geomechanics for impact on production. Rock Mechanics and Rock Engineering, 1-20 (DOI:10.1007/s00603-019-01892-8).

Yanlin, W., Bi Xiangjun, B., Sheng, F., Yingxin, M., and Qianjin, Y. [2011] Subsidence monitoring of offshore platforms. Procedia Engineering, 15, 1015-1020. 